\title{
Almost Unbiased Modified Linear Regression Estimators for Estimation of Population Mean
}

\section{J. Subramani and G. Kumarapandiyan}

\begin{abstract}
The present paper deals with a class of modified linear regression type estimators which are almost unbiased. We have derived their variances together with the values for which the proposed class of estimators perform better than the usual linear regression estimator. The performances of these proposed estimators are also assessed for certain natural populations available in the literature. It is observed from the numerical comparisons that the proposed estimators perform better than the existing modified ratio type estimators.
\end{abstract}

Keywords--- Auxiliary Variable, Modified Ratio Estimators, Natural Populations, Simple Random Sampling

\section{INTRODUCTION}

$\mathrm{I}^{\mathrm{N}}$ $\mathrm{N}$ sample surveys, auxiliary information on the finite population under study is quite often available from previous experience, census or administrative databases. The sampling theory describes a wide variety of techniques/ methods for using auxiliary information to improve the sampling design and to obtain more efficient estimators like Ratio, Product and Regression estimators. Ratio estimators, improves the precision of estimate of the population mean or total of a study variable by using prior information on auxiliary variable $X$ which is correlated with the study variable $Y$. Over the years the ratio method of estimation has been extensively used because of its intuitive appeal and the computational simplicity.

The classical Ratio estimator for the population mean $\bar{Y}$ of the study variable $Y$ is defined as:

$$
\hat{\bar{Y}}_{\mathrm{R}}=\frac{\bar{y}}{\bar{x}} \bar{X}=\widehat{R} \bar{X}, \text { where } \quad \widehat{R}=\frac{\bar{y}}{\bar{x}}=\frac{y}{x}
$$

where $\widehat{R}$ is the estimate of $R=\frac{\bar{Y}}{\bar{X}}=\frac{Y}{X}, \bar{y}$ is the sample mean of the study variable $Y$ and $\bar{x}$ is the sample mean of auxiliary variable $X$. It is assumed that the population mean $\bar{X}$ of auxiliary variable $X$ is known. The bias and mean squared error of $\hat{Y}_{\mathrm{R}}$ to the first degree of approximation are given below

$$
\begin{aligned}
& B\left(\hat{\bar{Y}}_{R}\right)=\frac{(1-f)}{n} \bar{Y}\left(C_{x}^{2}-C_{x} C_{y} \rho\right) \\
& \operatorname{MSE}\left(\hat{\bar{Y}}_{R}\right)=\frac{(1-f)}{n} \bar{Y}^{2}\left(C_{y}^{2}+C_{x}^{2}-2 C_{x} C_{y} \rho\right)
\end{aligned}
$$

J. Subramani, Associate Professor, Department of Statistics, Pondicherry University, Puducherry -605014, India.E-mail:drjsubramani@yahoo.co.in

G. Kumarapandiyan, Research Scholar, Department of Statistics, Pondicherry University, Puducherry-605014, India. E-mail: kumarstat88@gmail.com
The usual linear regression estimator together with variance is given as

$$
\begin{aligned}
& \hat{\bar{Y}}_{l r}=\bar{y}+\hat{\beta}(\bar{X}-\bar{x}) \\
& V\left(\hat{\bar{Y}}_{l r}\right)=\frac{(1-f)}{n} S_{y}^{2}\left(1-\rho^{2}\right)
\end{aligned}
$$

where $\hat{\beta}$ is the sample regression coefficient of $Y$ on $X$.

Many modified ratio type estimators available in the literature are biased but have minimum mean squared errors compared to that of usual ratio estimator. Some of the modified ratio estimators, which are to be compared with that of the proposed estimators, are listed below.

Sisodia and Dwivedi [2] has suggested a modified ratio estimator using the population coefficient of variation $C_{x}$ of auxiliary variable $X$ for estimating $\bar{Y}$ together with its bias and mean squared error and are as given below:

$\widehat{\bar{Y}}_{1}=\bar{y}\left[\frac{\bar{X}+C_{x}}{\bar{x}+C_{x}}\right]$

$B\left(\hat{\bar{Y}}_{1}\right)=\frac{(1-f)}{n} \bar{Y}\left(\theta_{1}^{2} C_{x}^{2}-\theta_{1} C_{x} C_{y} \rho\right)$

$\operatorname{MSE}\left(\hat{Y}_{1}\right)=\frac{(1-f)}{n} \bar{Y}^{2}\left(C_{y}^{2}+\theta_{1}^{2} C_{x}^{2}-2 \theta_{1} C_{x} C_{y} \rho\right)$

where $f=\frac{n}{N}$ and $\theta_{1}=\frac{\bar{X}}{\bar{X}+C_{x}}$

When the coefficient of kurtosis of auxiliary variable $X$ is known, Singh et.al [3] has developed a modified ratio type estimator for estimating $\bar{Y}$ and derived its bias and mean squared error as given below:

$\hat{\bar{Y}}_{2}=\bar{y}\left[\frac{\bar{X}+\beta_{2}}{\bar{x}+\beta_{2}}\right]$

$B\left(\hat{\bar{Y}}_{2}\right)=\frac{(1-f)}{n} \bar{Y}\left(\theta_{2}^{2} C_{x}^{2}-\theta_{2} C_{x} C_{y} \rho\right)$

$\operatorname{MSE}\left(\hat{\bar{Y}}_{2}\right)=\frac{(1-f)}{n} \bar{Y}^{2}\left(C_{y}^{2}+\theta_{2}^{2} C_{x}^{2}-2 \theta_{2} C_{x} C_{y} \rho\right)$

where $f=\frac{n}{N}$ and $\theta_{2}=\frac{\bar{X}}{\bar{X}+\beta_{2}}$

Motivated by Singh et.al [3], Yan and Tian [6] has suggested another modified ratio estimator using the coefficient of skewness of the auxiliary variable $X$ together with its bias and mean squared error and are as given below:

$\hat{\bar{Y}}_{3}=\bar{y}\left[\frac{\bar{X}+\beta_{1}}{\bar{x}+\beta_{1}}\right]$ 


$$
\begin{gathered}
B\left(\hat{Y}_{3}\right)=\frac{(1-f)}{n} \bar{Y}\left(\theta_{3}^{2} C_{x}^{2}-\theta_{3} C_{x} C_{y} \rho\right) \\
\operatorname{MSE}\left(\hat{\bar{Y}}_{3}\right)=\frac{(1-f)}{n} \bar{Y}^{2}\left(C_{y}^{2}+\theta_{3}^{2} C_{x}^{2}-2 \theta_{3} C_{x} C_{y} \rho\right) \\
\text { where } f=\frac{n}{N} \text { and } \theta_{3}=\frac{\bar{X}}{\bar{X}+\beta_{1}}
\end{gathered}
$$

When the population correlation coefficient between $X$ and $Y$ is known, Singh and Tailor [5] proposed another estimator for estimating $\bar{Y}$ together with its bias and mean squared error and are as given below:

$$
\begin{gathered}
\hat{Y}_{4}=\bar{y}\left[\frac{\bar{X}+\rho}{\bar{x}+\rho}\right] \\
B\left(\hat{Y}_{4}\right)=\frac{(1-f)}{n} \bar{Y}\left(\theta_{4}^{2} C_{x}^{2}-\theta_{4} C_{x} C_{y} \rho\right) \\
\operatorname{MSE}\left(\hat{\bar{Y}}_{4}\right)=\frac{(1-f)}{n} \bar{Y}^{2}\left(C_{y}^{2}+\theta_{4}^{2} C_{x}^{2}-2 \theta_{4} C_{x} C_{y} \rho\right) \\
\text { where } f=\frac{n}{N} \text { and } \theta_{4}=\frac{\bar{X}}{\bar{X}+\rho}
\end{gathered}
$$

By using the population variance of auxiliary variable $X$, Singh [4] proposed a modified ratio type estimator for estimating $\bar{Y}$ together with its bias and mean squared error and are as given below:

$\hat{\bar{Y}}_{5}=\bar{y}\left[\frac{\bar{X}+S_{x}}{\bar{x}+S_{x}}\right]$

$B\left(\hat{\bar{Y}}_{5}\right)=\frac{(1-f)}{n} \bar{Y}\left(\theta_{5}^{2} C_{x}^{2}-\theta_{5} C_{x} C_{y} \rho\right)$

$\operatorname{MSE}\left(\hat{\bar{Y}}_{5}\right)=\frac{(1-f)}{n} \bar{Y}^{2}\left(C_{y}^{2}+\theta_{5}^{2} C_{x}^{2}-2 \theta_{5} C_{x} C_{y} \rho\right)$

where $f=\frac{n}{N}$ and $\theta_{5}=\frac{\bar{X}}{\bar{X}+S_{x}}$

The estimators discussed above are biased but having minimum mean squared error compared to the usual ratio estimator. These points have motivated us to introduce a class of almost unbiased modified linear regression estimators. In fact the proposed estimators are all unbiased if the known population parameters are the true values. However in practical problems the known values are replaced by the values estimated from the previous studies or from another sample. Hence these values are not exactly equal to the true value of the population parameters. That is why the proposed estimators are called as almost unbiased modified linear regression estimators.

\section{PRoposed Modified LiNEAR REGRESSION ESTIMATORS}

New estimators are generally proposed or constructed by modifying the structure of the sampling designs or the structure of the estimators itself with reasonable and convincing motivations. Moving along this direction, we intend in this paper to show how the problem of estimating the unknown population mean of a study variable can be treated in a unified way by defining a class of estimators which will be (almost) unbiased and more efficient estimators.

The proposed modified linear regression estimators for population mean $\bar{Y}$ is

$$
\hat{\bar{Y}}_{N}=\bar{y}(1-\alpha)+\alpha \frac{s_{y}}{c_{y}}-(1-\alpha) \frac{s_{y}}{c_{y}} e_{1}
$$

where $e_{1}=\frac{\bar{x}-\bar{X}}{\bar{X}}$ and $\alpha$ is a suitably chosen scalar.

For the sake of convenience and to derive the variance, the proposed estimators given in (2.1) can be written in a more compact form as given below:

$$
\hat{\bar{Y}}_{N}=\bar{y}-S_{y}\left(\frac{\alpha}{c_{y}} e_{0}+\frac{(1-\alpha)}{c_{x}} e_{1}\right)
$$

where $e_{0}=\frac{\bar{y}-\bar{Y}}{\bar{Y}}$ and $S_{y}$ and $C_{y}$ are respectively the population standard deviation and coefficient of variation of the study variable $Y$ and $C_{x}$ is the co-efficient of variation of the auxiliary variable $X$. It is reasonable to assume that the values of $S_{y}$ and $C_{y}$ are known from the previous studies. Further we can write $\bar{y}=\bar{Y}\left(1+e_{0}\right)$ and $\bar{x}=\bar{X}\left(1+e_{1}\right)$ such that

$$
\begin{gathered}
E\left[e_{0}\right]=E\left[e_{1}\right]=0 \\
E\left[e_{0}^{2}\right]=\frac{(1-f)}{n} C_{y}^{2} \\
E\left[e_{1}^{2}\right]=\frac{(1-f)}{n} C_{x}^{2}, \\
E\left[e_{0} e_{1}\right]=\frac{(1-f)}{n} \rho C_{y} C_{x} \text { and } f=\frac{n}{N}
\end{gathered}
$$

Taking expectation on both sides of equation (12), the expected value of the proposed estimators are obtained as

$$
\begin{gathered}
E\left(\hat{\bar{Y}}_{N}\right)=E\left(\bar{y}-S_{y}\left(\frac{\alpha}{c_{y}} e_{0}+\frac{(1-\alpha)}{c_{x}} e_{1}\right)\right)=\bar{Y}, \text { since } E\left(e_{0}\right)= \\
E\left(e_{1}\right)=0
\end{gathered}
$$

From (14) we observe that proposed estimators are unbiased estimators. However the known values of $S_{y}, C_{y}$ ad $C_{x}$ may not be the same to their true values. Hence the proposed estimators are called as almost unbiased estimators. The corresponding variances of the proposed estimators are as given below:

$$
V\left(\hat{\bar{Y}}_{N}\right)=\frac{2(1-f)}{n} S_{y}^{2}(1-\alpha)^{2}(1-\rho)
$$

\section{EFFICIENCY OF THE PROPOSED ESTIMATORS}

For want of space and for the convenience of the readers, the mean squared errors discussed in equations (6) to (10) are represented in a class of modified ratio estimators as given below:

$\operatorname{MSE}\left(\hat{\bar{Y}}_{i}\right)=\frac{(1-f)}{n} \bar{Y}^{2}\left(C_{y}^{2}+\theta_{i}^{2} C_{x}^{2}-2 \theta_{i} C_{x} C_{y} \rho\right) ;$

$i=1,2,3,4,5$ 
where $f=\frac{n}{N}, \theta_{1}=\frac{\bar{X}}{\bar{X}+C_{x}}, \theta_{2}=\frac{\bar{X}}{\bar{X}+\beta_{2}}, \theta_{3}=\frac{\bar{X}}{\bar{X}+\beta_{1}}$,

$\theta_{4}=\frac{\bar{X}}{\bar{X}+\rho}$ and $\theta_{5}=\frac{\bar{X}}{\bar{X}+S_{x}}$

As mentioned earlier the variance of usual linear regression estimator is given as

$$
V\left(\hat{\bar{Y}}_{l r}\right)=\frac{(1-f)}{n} S_{y}^{2}\left(1-\rho^{2}\right)
$$

The variances of the proposed estimators are given as

$$
V\left(\hat{\bar{Y}}_{N}\right)=\frac{2(1-f)}{n} S_{y}^{2}(1-\alpha)^{2}(1-\rho)
$$

At various values of $\alpha$, variances of the proposed estimator are listed in the following table:

Table 1: Variances of the Proposed Estimators at Various Values of $\alpha$

\begin{tabular}{|c|c|}
\hline$\alpha_{i}$ & \multicolumn{1}{|c|}{$V\left(\hat{\bar{Y}}_{N}\right)$} \\
\hline$\alpha_{1}=1 \pm \sqrt{\frac{1}{2}}$ & $\frac{(1-f)}{n} S_{y}^{2}(1-\rho)$ \\
\hline$\alpha_{2}=1 \pm \frac{1}{S_{y}} \sqrt{\frac{1}{2}\left(\frac{n}{(1-f)}\right)}$ & $(1-\rho)$ \\
\hline$\alpha_{3}=1 \pm \frac{1}{S_{y}} \sqrt{\frac{1}{2}}$ & $\frac{(1-f)}{n}(1-\rho)$ \\
\hline
\end{tabular}

From the expressions given in (16) and (18) we have derived the conditions for which the proposed estimators $\hat{\bar{Y}}_{N}$ are more efficient than that of existing modified ratio estimators $\hat{Y}_{i} ; i=1,2,3,4$ and 5 are given below:

That is, $V\left(\hat{\bar{Y}}_{N}\right)<M S E\left(\hat{\bar{Y}}_{i}\right)$ if

$1-\sqrt{\frac{1+C_{y}^{2}\left(\theta_{i}^{2} C_{x}^{2}-2 \theta_{i} C_{x} C_{y} \rho\right)}{2(1-\rho)}}<\alpha<1+\sqrt{\frac{1+C_{y}^{2}\left(\theta_{i}^{2} C_{x}^{2}-2 \theta_{i} C_{x} C_{y} \rho\right)}{2(1-\rho)}}$

From the expressions given in (17) and (18) we have derived the conditions for which the proposed estimators $\hat{\bar{Y}}_{N}$ are more efficient than the usual linear regression estimator and are given below.

That is, $V\left(\hat{\bar{Y}}_{N}\right)<V\left(\hat{\bar{Y}}_{l r}\right)$

$$
\text { if } 1-\sqrt{\frac{1}{2}(1+\rho)}<\alpha<1+\sqrt{\frac{1}{2}(1+\rho)}
$$

\section{NUMERICAL STUDY}

The performances of the proposed estimators are assessed with that of the modified ratio estimators for three populations. The populations 1 and 2 are taken from Murthy [1] in page 228 and population 3 is taken from Murthy [1] in page 422 . The population constants obtained from the above data are given below:

Population 1: Murthy 1967 page 228 [1]

$\mathrm{X}=$ Fixed Capital and $\mathrm{Y}=$ Output for 80 factories in a region

$$
\mathrm{N}=80 \quad \mathrm{n}=20 \quad \overline{\mathrm{Y}}=51.8264 \quad \overline{\mathrm{X}}=11.2646
$$

$$
\begin{aligned}
& \rho=0.9413 \quad S_{y}=18.3569 \quad C_{y}=0.3542 \quad S_{x}=8.4563 \\
& C_{x}=0.7507
\end{aligned}
$$

Population 2: Murthy 1967 page 228 [1]

$\mathrm{X}=$ Data on number of workers and $\mathrm{Y}=$ Output for 80 factories in a region

$$
\begin{array}{llll}
\mathrm{N}=80 & \mathrm{n}=20 & \overline{\mathrm{Y}}=51.8264 & \overline{\mathrm{X}}=2.8513 \\
\rho=0.9150 & S_{y}=18.3569 & C_{y}=0.3542 & S_{x}=2.7042 \\
C_{x}=0.9484 & & &
\end{array}
$$

Population 3: Murthy 1967 page 422 [1]

Number of Cattle for 24 sample villages

\begin{tabular}{|c|c|c|c|c|}
\hline \multirow{3}{*}{\multicolumn{2}{|c|}{ Estimators }} & \multirow{2}{*}{\multicolumn{3}{|c|}{$\frac{\text { Mean squared errors }}{\text { Populations }}$}} \\
\hline & & & & \\
\hline & & 1 & 2 & 3 \\
\hline \multicolumn{2}{|l|}{$\widehat{\hat{\bar{Y}}_{1}}$} & 15.2581 & 17.1881 & 933.5226 \\
\hline \multicolumn{2}{|l|}{$\hat{\bar{Y}}_{2}$} & 19.3382 & 12.8425 & 929.2689 \\
\hline \multicolumn{2}{|l|}{$\hat{\bar{Y}}_{3}$} & 14.0112 & 21.3660 & 931.1454 \\
\hline \multicolumn{2}{|l|}{$\hat{\bar{Y}}_{4}$} & 14.4502 & 17.6849 & 933.4261 \\
\hline \multicolumn{2}{|l|}{$\hat{\hat{\bar{Y}}_{5}}$} & 2.3565 & 4.7220 & 2721.5855 \\
\hline \multicolumn{2}{|c|}{$\hat{\bar{Y}}_{l r}$} & 1.4399 & 2.0569 & 859.2285 \\
\hline \multirow{3}{*}{$\hat{\bar{Y}}_{N}$} & $\alpha_{1}$ & 0.7418 & 1.0741 & 438.6280 \\
\hline & $\alpha_{2}$ & 0.0587 & 0.0850 & 0.0411 \\
\hline & $\alpha_{3}$ & 0.0022 & 0.0031 & 0.0017 \\
\hline
\end{tabular}

$$
\begin{array}{clll}
\text { X- Census } & \multicolumn{1}{c}{\text { Y-Survey }} & & \\
\mathrm{N}=24 & \mathrm{n}=12 & \overline{\mathrm{Y}}=568.5833 & \overline{\mathrm{X}}=568.25 \\
\rho=0.9589 & S_{y}=506.1174 & C_{y}=0.8901 & S_{x}=528.0501 \\
C_{x}=0.9293 & & &
\end{array}
$$

The mean squared errors of the existing modified ratio estimators and the variance of the proposed estimators (at different values of $\alpha$ ) and linear regression estimators for the three populations discussed above are given in the following table:

Table 2: Mean Squared Errors of the Existing Modified Ratio estimators, Linear Regression and Proposed Estimators

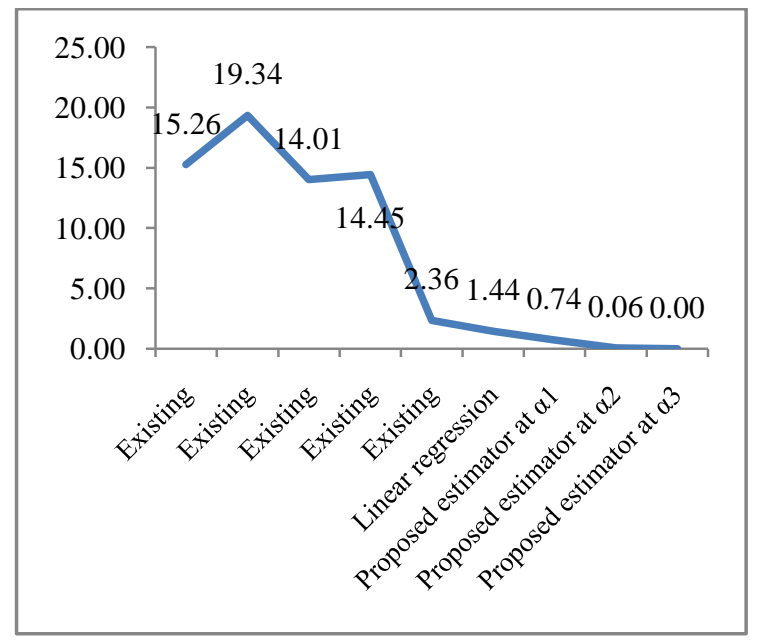

Figure 1: Mean Squared Errors of the Existing, Linear Regression and Proposed Estimators for Population 1 


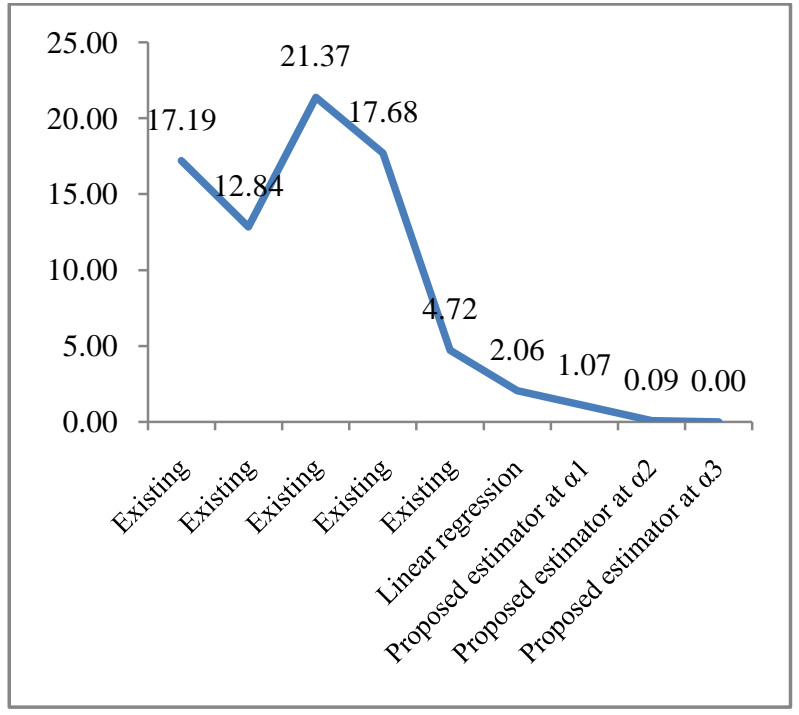

Figure 2: Mean Squared Errors of the Existing, Linear Regression and Proposed Estimators for Population 2

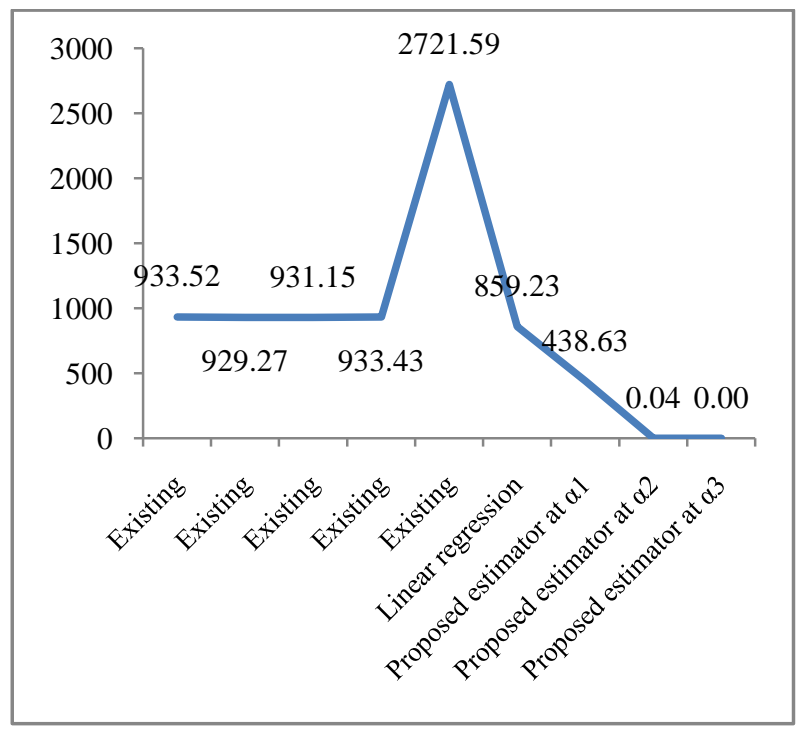

Figure 3: Mean Squared Errors of the Existing, Linear Regression and Proposed Estimators for Population 3

\section{CONCLUSION}

In this paper we have suggested modified linear regression type estimators and also obtained their variances. Further we have also derived the conditions for which proposed estimators perform better than the existing modified ratio and linear regression estimators. We have also assessed the performances of the proposed estimators for certain known populations. From the numerical comparisons, we have observed that the proposed estimators are performed better than that of modified ratio type estimators and linear regression estimator.

\section{ACKNOWLEDGEMENT}

The second Author wishes to record his gratitude and thanks to the Vice Chancellor, Pondicherry University and other University authorities for being given me the Financial
Assistance to carry out this research work through the University Fellowship.

\section{REFERENCES}

[1] M.N. Murthy, "Sampling theory and methods", Statistical Publishing Society, Calcutta, India, 1967.

[2] B.V.S. Sisodia and V.K. Dwivedi, "A modified ratio estimator using coefficient of variation of auxiliary variable", Jour. Ind. Soc. Agri. Stat., Vol. 33(1), Pp. 13-18, 1981.

[3] H.P. Singh, R. Tailor, R. Tailor and M.S. Kakran, "An Improved Estimator of population mean using Power transformation", Journal of the Indian Society of Agricultural Statistics, Vol. 58(2), Pp. 223-230, 2004.

[4] G.N. Singh, "On the improvement of product method of estimation in sample surveys", Jour.Ind. Soc. Agri. Statistics, Vol. 56 (3), Pp. 267$265,2003$.

[5] H.P. Singh and R. Tailor, "Use of known correlation coefficient in estimating the finite population means", Statistics in Transition, Vol. 6 (4), Pp. 555-560, 2003.

[6] Z. Yan and B. Tian, "Ratio Method to the Mean Estimation Using Coefficient of Skewness of Auxiliary Variable", ICICA 2010, Part II, CCIS 106, Pp. 103-110, 2010.

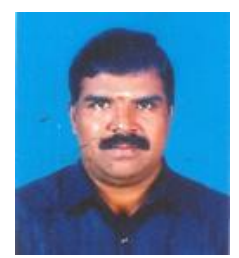

Dr. J. Subramani has received the doctorate degree in Statistics from University of Madras. He is currently working as Associate Professor, Department of Statistics, Pondicherry University. He has more than 25 years of experience both in Teaching and Research. To his academic credit he has received U.S.Nair Young Statistician Award from Indian Society of Probability and Statistics and also the International Young Statistician Award from the International Statistical Institute, The Netherlands. His research interests are Estimation of Variance Components, Missing Data Analysis, Sampling Theory, Incomplete Block Designs, Control Charts and Process Capability Analysis. He has published more than 75 research papers in reputed International and National Journals. He has also participated in many conferences and workshops in India as well as abroad and presented research papers; delivered invited talks and special addresses. He has organized many International and National Cosnferences and also acted as an organizing committee member in many conferences.

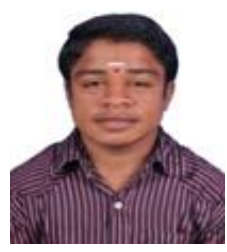

G. Kumarapandiyan did his M.Sc. Statistics with First class distinction at Department of Statistics, Ramanujan School of Mathematical Sciences, Pondicherry University, R.V. Nagar, Kalapet, Puducherry-605 014 during 2009-2011. He is presently pursuing his Ph.D. in Statistics in the area of Sampling Theory under the guidance of Dr. J. Subramani, Associate Professor, Department of Statistics, Pondicherry University, Puducherry. He has been awarded the "Pondicherry University Gold medal" for best performance in B.Sc. Statistics, "Shri Shankar Dayal Sharma Gold Medal" for the best performance in M.Sc. Statistics and also the "Vijaya Bank Gold Medal" for the Best PG Student of Pondicherry University. 